\title{
Technology and Public Access to Cultural Heritage: The Italian Experience on ICT for Public Historical Archives
}

\author{
Calogero Guccio, Marco Ferdinando Martorana, Isidoro Mazza, \\ and Ilde Rizzo
}

\begin{abstract}
The introduction and diffusion of digital technologies have had a tremendous impact on the production, preservation and utilisation of cultural heritage. In Italy, the Ministry for Cultural Heritage and Activities and Tourism (MiBACT) has undertaken several programs involving the use of digital technology to promote a larger access to cultural heritage, through the collection of metadata on cultural products preserved in the country and the provision of digital cultural products. Digitisation techniques and web infrastructures affect most activities carried out by such institutions: the production of cultural goods, the use and valorisation of cultural heritage, as well as the costs of preservation. This study analyses the digital projects carried out by the MiBACT for the preservation and utilisation of cultural heritage that is managed by public historic archives so as to evaluate their impact on the access to cultural products.
\end{abstract}

\section{Introduction}

Digital technologies have determined a rapid and substantial change in the practices of utilisation, supply, and conservation of cultural heritage. Some studies analysed the general impact of digitisation on cultural policy (see Flew and Swift 2013), and on museums and libraries in particular (Navarrete 2013a, b; Paolini et al. 2013; Salaün 2013). This blooming literature, however, has so far neglected, with a few exceptions (Borowiecki and Navarrete 2015), to investigate the implications of digitisation for public archives that store and preserve cultural heritage.

\footnotetext{
C. Guccio $(\bowtie) \cdot$ M.F. Martorana • I. Mazza • I. Rizzo

Department of Economics and Business, University of Catania, Corso Italia, 55, Catania 95128, Italy

e-mail: guccio@unict.it; marco.martorana@unict.it; imazza@unict.it; rizzor@unict.it
} 
From a theoretical point of view, digitisation techniques and web infrastructures affect all activities carried out by such institutions. Firstly, digitisation stimulates the production of cultural goods. Secondly, management and valorisation may improve, since institutions may easily handle acquisition, exchange and exhibition of products through digital catalogues, while a single web portal collecting metadata on the country's cultural heritage may help its promotion. Lastly, digitisation of cultural goods combined with the spread of web connections reduce access costs and overcome geographical and time constraints.

This chapter studies the extent to which the introduction of digital technology affects the production, valorisation and utilisation of cultural heritage existing in public historical archives in Italy, comparing it to its European counterparts from theoretical and empirical perspectives. With this aim, we study the actual extent to which European and national level projects are involving the use of digital technologies, with specific attention to the degree of digital indexing, digitisation, and the use of internet websites. We find that the introduction of different digital technologies occurs only partially in parallel, that is, more complex procedures are introduced only after the basic ones. Although digital indexing has substantially spread, digitisation is at an initial stage and the same can be said about digital access. This is true for the EU as well as Italy, which is also characterized by persistent geographical differences across its regions. In addition, the full implementation of websites seems to have no relevant effects on physical access. The chapter is organised as follows. In Sect. 2, we analyse theoretical aspects related to the introduction of digital technologies in the preservation and utilisation of cultural objects. Section 3 provides a general review of the digital projects carried out so far at European and Italian levels and analyses the current scope of these projects. Section 4 focuses on Italian public historical archives and includes an extensive analysis of the magnitude of digital projects in Italy. Some comments conclude the chapter.

\section{Economic Implications of Digitisation}

\subsection{Digital Heritage}

Digitisation implies the adoption of technology to store and transfer content. It therefore influences considerably the costs of access and preservation. This circumstance is particularly relevant for cultural heritage where digitisation means making heritage objects and services digital. As for the objects, such a process entails some form of representation (or visualization) as well as description (or contextualization); thus, digitisation of heritage refers to the 'object' as well as to its documentation.

In the literature, a wide definition of digital heritage goods is provided. For instance, according to Navarrete (2013a), we can identify three types of digital heritage goods: digitised goods, metadata and born-digital goods. Digitisation usually refers to the generation of a copy of a physical original, e.g. the scan of an 
archival document or the digital image of a painting. The digitisation of information (such as size, date, origin, title, description, context) resulting from earlier documentation (e.g. paper archive, object registration cards) or from personal knowledge generated metadata which are useful to identify, describe, understand and value heritage objects. In other cases, for instance, digital (video) art, content is generated in digital form from the beginning, e.g. born-digital goods. To investigate the economic implications of digitisation, it is important to recall that heritage objects can be movable and immovable, tangible and intangible and housed in different type of cultural institutions such as archives, libraries, museums, historical buildings or archaeology sites. As described further in detail, these differences are bound to influence the effects of digitisation on the supply and demand of heritage.

\subsection{Supply and Demand of Heritage}

Digitisation affects the supply and demand of heritage and the economic nature of heritage goods and services, since it influences two crucial economic characteristics of their consumption: rivalness and excludability. ${ }^{1}$

The effects of digitisation differ substantially depending on the heritage item. For libraries and archives, access to hard copies of books and documents is fully rival, while in the case of museums, historical buildings or archaeological sites rivalness occurs only in case of congestion and, therefore, it hardly emerges in the less popular heritage. Thus, for the first category of goods, digitisation allows for joint consumption, also when this would not be possible for the original items. ${ }^{2}$

From a different perspective, the application of technology might be helpful in reducing the conflict between the objectives of preservation vs. utilisation. ${ }^{3}$ In other words, technology generates positive effects on the sustainability of heritage. At a site with problems of extreme decay and deterioration, virtual visits can substitute real ones. Of course, this also applies to archives especially when very old paper documents are involved and their inspection is very risky. Indeed, in the case of extreme decay, which would prevent usage anyway, digitisation generates private benefits, which would not occur otherwise because of the risks connected to the direct use of the item.

The digital access to heritage sites is generally more public than the 'real' one. In fact, even though web access could be easily restricted technically, the large availability of images and information on the web makes such limitation pointless in many instances. Moreover, a decision to limit access (for example, making it on-demand) may well contrast with the institutional mission of museums or

\footnotetext{
${ }^{1}$ More in general, the effects of technology on the demand and supply of heritage goods are investigated by Giardina et al. (2015).

${ }^{2}$ However, digitisation lowers the access cost as it can be accessed from remote location.

${ }^{3}$ An interesting example is the Mayan archaeological site of Calakmul in Mexico, which UNESCO declared as a World Heritage site in 2002 (Peacock and Rizzo 2008).
} 
archives for open access. Websites of those cultural institutions have the goal of enlarging the number of users, allowing anyone to visit virtually while being at home, expanding the range of sources of information about heritage, increasing consumers' knowledge and, therefore, improving their critical appraisal.

Differences occur across different institutions also in relation to the distinctiveness and costs of the service. The digital service is commonly directed to satisfy a demand for 'virtual' visits in the form of entertainment. On the contrary, a specific demand that asks for a high standard of precision, completeness and swiftness, coming from researchers or professionals, may induce price exclusion. This occurrence may be more frequent in case of archives or libraries. For instance, Navarrete (2013a) recalls that the city of Amsterdam's archive offers digitisation on demand and charges a higher price for higher image resolution, a rush fee for processing requests in less than 2 weeks and a fee for access from home.

Digitisation, then, broadens the set of users but also causes an overlapping supply of two rather different cultural good or service, of 'hard' (real) and 'digital' kind. This phenomenon raises the question whether digitisation exerts either a substitution effect on real visits or a complementary one. This question has no univocal answer, as it very much depends on the type of good under consideration. After all, the enjoyment deriving from the real experience of visiting a museum or a heritage site can hardly be substituted by a digital copy of a painting or by a virtual tour. Therefore a relationship of complementarity between the 'hard' and 'digital' is more likely to arise. ${ }^{4} \mathrm{~A}$ rather different situation emerges in the case of other cultural institutions such as archives or libraries. Access to a digitised document may be understood as more equivalent to the vision of the original document, depending on the quality of the digitisation and the goals of the research. However, it is worth mentioning that the use of 'virtuality' as a tool for the valorisation of heritage is not unanimously accepted by experts who claim that it might downgrade the 'high' character of heritage.

\subsection{The Case of Public Archives}

In general, we could say that digital environment enhances the economic potentialities of the cultural sector. Bakhshi and Throsby (2012) emphasize the creation of new and diversified cultural products, the development of new cultural heritage experiences. The digital world improves the possibilities of contextualising cultural heritage, which has always been important for understanding its impact. Technology makes this contextualizing easier and wider in scope. Furthermore, the availability of metadata allows users to create their own virtual collection and learn the stories related to the items. In addition, other benefits arise from knowledge

\footnotetext{
${ }^{4}$ In presence of visits motivated by entertainment, Peacock (2006: 1138) argues that technological changes are likely to create a 'globalization of culture', generating international mobility of artistic production and exhibition, as well as of tourists and increasing the demand for heritage.
} 
transfers and from a technologically dynamic creative economy. For example, some museums, such as the Metropolitan Museum of Art in New York or the Rijksmuseum in Amsterdam, provide open access to content (text, video, photo, music) generated by museum visitors in social networks, encouraging exchanges and communication among people. As Clough (2013) suggests, cultural institutions also face a big opportunity, using their content and new technologies to reduce the increasing disparity between the educational opportunities available to children in upper income groups and those of lower income groups.

This brief analysis suggests that archives are the form of cultural heritage that is likely to benefit most from digitisation for several reasons. Leaving aside the benefits deriving from the improvement in preservation and the reduction of costs for maintenance (which have to outweigh the costs of digitisation), which are fairly common issues for all forms of cultural heritage although with a different scope, there are some matters that distinguish public archives from others in terms of digitisation. First, regarding the consumption of their services, digitisation transforms a substantially private service (rival and excludible) into a collective one available to anyone at the same time. A digitised archive then requires the application of different efficiency conditions with respect to its 'real' counterpart. Second, an archive is likely to be used by experts, such as researchers and professionals. They may however have different expectations about the quality of the digitised documents. A lawyer, for example, may be interested in the pure content of the text, whereas a researcher may also be interested in a detailed highquality reproduction of the whole document. This suggests that, digitisation allows for product differentiation, with more definite images available upon request. Finally, the problem of the prevalence between substitution and complementary effects is somewhat more marginal for the archives than for the contents of museums or archaeological sites. In fact, this problem is practically non-existent for those who are concerned just with the content of the text. A digital copy is fully equivalent to the original for their purposes, whereas it may be relevant for the usage of images contained in the document. On the one hand, the original prevails for the more comprehensive enjoyment of the artwork; on other hand, the intelligibility of small miniatures is improved by a digital image able to magnify small details.

\section{Digital Projects on Cultural Heritage: An Overview}

\subsection{Background}

After having highlighted some theoretical issues concerning the impact of digitisation on the supply, utilisation and conservation of cultural heritage, especially for the case of public archives, this section reviews the main digital projects in Europe and Italy that are related to the issues investigated here. Digital technologies have become increasingly important in the field of preservation and utilisation of cultural goods. Recently, the EU has undertaken several projects involving the application of such technologies, which include the digitisation of 
tangible and intangible cultural heritage and the use of Information and Communication Technologies (ICT) to improve: the conservation and preservation of cultural products; the digital and physical access as well as tourism; and the management of heritage throughout Europe. Following this example, many countries have adopted formal strategies and new practises to enhance the use of new technologies and, as far as Italy is concerned, the MiBACT introduced several programs accordingly. In this Section, we provide a brief overview of these projects, starting at European level programs, and show their state of the art, including details on the degree of digitisation, with a specific focus on Public Historical Archives (PHAs).

\subsection{European Projects}

By the end of the 1990s, the use of digital technologies to cultural heritage has spread in Europe and has resulted in several projects developed at national and continental levels. The European Library (2005) represented the first large program involving the collection of metadata belonging to several institutions (national libraries) across Europe. Following that, in 2008, the European Commission launched the first version of Europeana, the internet portal collecting metadata on cultural heritage preserved by several institutions.

Europeana aims at enhancing the spread of culture throughout Europe by storing in a single portal all the contextual information related to the cultural products preserved by all its cultural institutions. The ambition is to allow the public (i.e. students, researchers, tourists, etc.) to easily find any item they are searching, and to promote programs of digitisation of cultural resources. The process of digital indexing and metadata production moves from cultural institutions, which in turn provide such data to Europeana, and it is currently far from being complete. Yet, the portal provides access to about 40 million digitised items of different types, including images, text, audio, and 3D files from all European countries. Since digitisation procedures are not straightforward, international standards have been applied to have homogeneous metadata, thus forcing institutions to use common procedures. Moreover, Europeana uses the Linked Open Data (LOD) paradigm, a technique for publishing data on the internet that allows to connect related data and make them freely accessible. ${ }^{5}$ Through digital projects such as Europeana, the EU aims at promoting universal access to cultural heritage, ${ }^{6}$ leading providers of cultural goods across Europe to change their practices according to international standards for data indexing and storage.

\footnotetext{
${ }^{5}$ This is in line with European Commission Recommendation of 27 October 2011 'on the digitisation and online accessibility of cultural material and digital preservation', which stresses the importance of re-using digitised material as a tool for economic and cultural development in the EU.

${ }^{6}$ See on this point the European Commission Recommendation of 27 October 2011.
} 


\subsection{Italian Projects}

In line with the above mentioned European programs, several projects have been carried out in Italy by the MiBACT, involving the use of ICT to improve the management of public institutions devoted to the preservation and conservation of cultural products and lessening the digital divide across cultural institutions within the country, ${ }^{7}$ and favour the utilisation of cultural products by the public. Such projects include the introduction of common procedures for information technology management; the use by the MiBACT and other cultural institutions of website and social media to facilitate and promote cultural events, the physical and digital access to cultural products as well as tourism; the digitisation of tangible and intangible heritage and the production of new digital products; the use of digital technologies (such as photo stitching and time lapse) to create digital representation of cultural sites to be browsed online; and the creation of national aggregators, in line with the abovementioned Europeana. ${ }^{8}$

In 2008, the MiBACT launched the Culturaltalia portal, which is held by the Union Catalogue of Italian Libraries (ICCU). CulturaItalia is integrated in Europeana, following the same mission at the national level: it aims at promoting Italian cultural heritage, providing a virtual access point to all the cultural products held by Italian institutions, and enhancing the process of digitisation of cultural resources. It is a national aggregator, which includes about 2.5 million items from 32 public and private partners, including other aggregators, as well as editorial articles where items, collections, cultural events and providers are described (Caffo 2014). It is an 'open' system since partners continuously upload digitised products which are in turn exported into Europeana (Di Giorgio 2014). Following the LOD paradigm, metadata is also available through a data management project run in 2012, the dati.culturaitalia.it, which is still under development, and includes metadata from a selected number of providers associated to Culturaltalia. ${ }^{9}$ As well as its continental level counterpart, Europeana, the amount of available resources depends on indexing and digitisation procedures run by its thematic partners and cultural institutions that own the original items. So far the extent of metadata provided by CulturaItalia is rather limited compared with the original ambitions of the project.

\footnotetext{
${ }^{7}$ In general terms, digital divide is the structural geographical difference in the use of digital technologies both on the supply and demand. Evidence of such a phenomenon within Europe and Italy, can be found in Vicente and Lopez (2011).

${ }^{8} \mathrm{~A}$ comprehensive overview of such projects, including related links to all the programs can be found in MiBACT (2015).

${ }^{9}$ Other relevant related programs are: the Internet Culturale (IC), a web portal, online since 2005, held by Union Catalogue of Italian Libraries (ICCU), which provides access to digital material and catalogue databases from Italian libraries and other relevant cultural institutions; and MuseiDItalia program, which aims at building an analogous portal including metadata on Italian museums. All these projects are, in turn, integrated in the national and European level aggregators, CulturaItalia and Europeana.
} 
In this chapter, we focus attention on Italian Public Historical Archives (PHAs). According to the latest edition of the Culture in Italy basic figures 2014 (MiBACT, 2014), the annual report of summary statistics on cultural utilisation and preservation in Italy, archivist institutions in Italy include: 100 PHAs, one Central State Archive and other 34 historical archives under the MiBACT, 8250 local authorities archives, about 50,000 other archives held by public institutions and 4609 statecontrolled private archives.

PHAs preserve 1,352,185 parchments and 13,805,410 folders, volumes, registers, etc. To promote the digitisation of such a robust quantity of cultural heritage and the digital access to the products conserved by all archivist institutions, the MiBACT supported the creation of state archives websites, which have been gathered in the MiBACT web-domain (beniculturali.it). It also established the Central Institute of Archives (ICAR), which is devoted to the management, development and harvesting of the archival information systems and run the National Archivist System (SAN), a national web aggregator which collects metadata in line with the abovementioned European protocols and is integrated within the national aggregator Culturaltalia, the European archivist aggregator Archives Portal Europe (APEx) and Europeana. ${ }^{10}$ The SAN is an open system which is uploaded as soon as the indexing and digitisation of cultural resources carried on by any archivist institutions progress. PHAs represent the most relevant sources of the whole archivist heritage and in recent times have been driven to improve their practices moving towards the use of digital technologies. They have been compelled to create and hold their websites, within the MiBACT's domain, and to proceed with the digitisation of the documents that they preserve. The progress of such new practices is still heterogeneous. While almost all the PHAs run a website, which include basic information such as opening times, and a list of provided services, the digitisation process is still at the beginning. The next section provides an overview of digitisation programs in cultural institutions in Europe and Italy with a specific focus on PHAs (Fig. 1).

\subsection{Digital Projects for Public Historical Archives}

We draw data from Enumerate Core Survey 3, a database founded by the European Commission to collect data on digitisation programs, digital preservation and digital access to cultural heritage in Europe, to compare the extent of digitisation

\footnotetext{
${ }^{10}$ The SAN includes about 800,000 archivist resources, It was been instituted in 2011 in order to: (i) offer a unique online access point to the Italian archivist heritage and a digital library, which provide digital products and all the metadata; (ii) make available to the general public complete information on the cultural products held by archives, on their producers and providers as well as on their accessibility; (iii) guarantee the use of common protocols for indexing, description and photographic reproduction of cultural products; (iv) produce integrate archivist thematic portals and the harvesting of all the archivist systems.
} 


\section{Italian aggregators for Europeana}

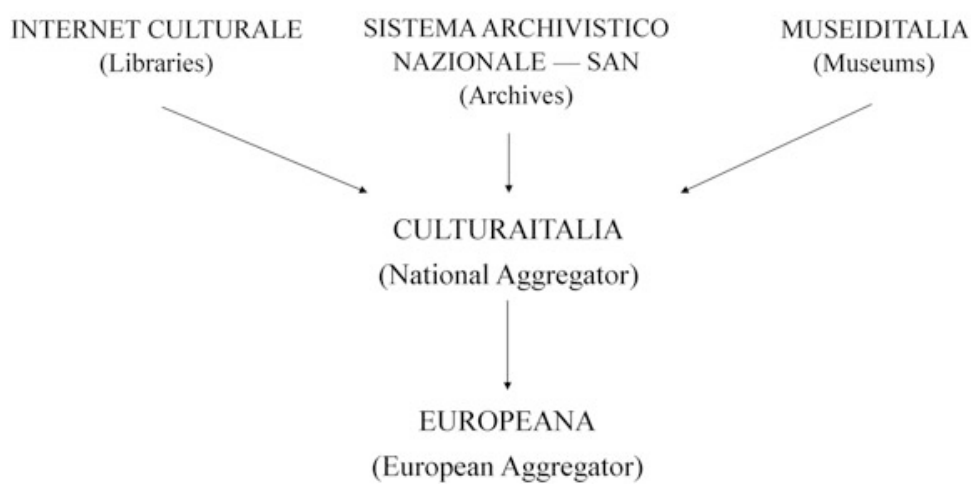

Fig. 1 Visual representation of Italian aggregators. Notes: IC stands for Internet Culturale, the librarian resources aggregator, SAN is the archives' resources aggregator and Museid Italia is the aggregator for museums' resources

in Italy and in EU. ${ }^{11}$ The dataset suffers from missing values and the sample itself is not representative, thus findings reported in the next sections have to be considered cautiously. Moreover, there are no available data for several countries with respect to archives. In what follows we consider the subsample of those countries for which there are at least two archives in the sample.

Sixty percent of the institutions collect born digital material, while this percentage was barely above $50 \%$ in the two previous surveys. The survey also included information on digital access. It emerges that web statistics are the primary means used by institutions to monitor the access to their metadata and digital objects. Table 1 shows the average data for all of the sample and the subsample of archives and allow us to draw some preliminary insights in a comparative perspective on the use of digital technologies and, more in particular, on digital indexing (which is connected to the development of Europeana and parallel national level projects) and digitisation. On average, the $58 \%$ of collections has been digitally catalogued. Moreover, only the $22 \%$ (12\% in the subsample of archives) of collections have

\footnotetext{
${ }^{11}$ More in depth, Enumerate Core Survey 3 is the third edition of a European survey monitoring the status of cultural heritage in Europe. One thousand and thirty institutions belonging to 32 European countries participated to this third round (participants to Core Survey 2 are about 1400). The dataset includes information for each institution in 2015 with respect to: the state of digitisation activity, the dimension and characteristics of collections, digital access, preservation strategy and expenditure. Institutions are distinguished in four types (Museum, $34.47 \%$; library, $33.59 \%$; Archive/record office, $21.12 \%$; other type, $10.78 \%$ ). Almost all institutions have collections to be preserved and $84 \%$ have a digital collection (this percentage was $83 \%$ in Core Survey 1 and $87 \%$ in Core Survey 2). See Stroeker and Vogels (2014) and Nauta \& van den Heuvel (2015) for a detailed analysis on the extent of digitisation in Europe and on latest versions of Enumerate Core Survey.
} 
Table 1 Impact of digitization on archives

\begin{tabular}{|c|c|c|c|c|c|c|}
\hline \multirow[b]{2}{*}{ Country } & \multicolumn{2}{|c|}{$\begin{array}{l}\text { Collection already } \\
\text { indexed }(\%)\end{array}$} & \multicolumn{2}{|c|}{$\begin{array}{l}\text { Collection already } \\
\text { digitised }(\%)\end{array}$} & \multicolumn{2}{|c|}{$\begin{array}{l}\text { Collection to be } \\
\text { digitised }(\%)\end{array}$} \\
\hline & All sample & Archives & All sample & Archives & All sample & Archives \\
\hline Austria & 60.15 & 50.63 & 24.46 & 27.63 & 49.15 & 38.00 \\
\hline Belgium & 64.29 & 56.67 & 23.86 & 5.67 & 45.00 & 25.00 \\
\hline $\begin{array}{l}\text { Czech } \\
\text { Republic }\end{array}$ & 69.29 & 57.50 & 22.86 & 22.50 & 49.29 & 42.50 \\
\hline Estonia & 74.00 & 71.50 & 15.89 & 10.75 & 65.44 & 71.50 \\
\hline Finland & 53.77 & 64.60 & 28.60 & 45.00 & 36.33 & 16.40 \\
\hline Germany & 51.29 & 55.11 & 15.71 & 14.05 & 39.54 & 33.84 \\
\hline Hungary & 47.91 & 15.00 & 13.87 & 2.00 & 44.09 & 25.60 \\
\hline Iceland & 57.63 & 50.00 & 24.63 & 20.00 & 56.44 & 36.40 \\
\hline Italy & 54.95 & 54.00 & 31.50 & 11.50 & 45.21 & 63.50 \\
\hline Lithuania & 19.82 & 22.00 & 15.19 & 2.88 & 67.91 & 70.13 \\
\hline Netherlands & 75.30 & 72.67 & 29.74 & 8.87 & 41.70 & 31.77 \\
\hline Portugal & 56.12 & 49.00 & 20.64 & 13.86 & 71.22 & 83.83 \\
\hline Slovenia & 61.82 & 51.25 & 19.98 & 2.00 & 50.31 & 16.25 \\
\hline Spain & 63.35 & 56.42 & 27.06 & 16.78 & 51.39 & 63.78 \\
\hline Sweden & 47.83 & 48.75 & 14.97 & 8.00 & 52.70 & 44.15 \\
\hline Switzerland & 70.29 & 63.33 & 17.90 & 4.67 & 35.15 & 31.50 \\
\hline $\begin{array}{l}\text { Sample } \\
\text { average }\end{array}$ & 58.29 & 55.00 & 22.85 & 12.81 & 48.98 & 45.45 \\
\hline
\end{tabular}

National level average-year 2015

Source: Enumerate Core Survey 3

been digitised so far and more than $49 \%$ of preserved heritage has to be digitised. Thus, in spite of the several projects, the digitisation process is still in its early stages and its scope is heterogeneous, ranging between 2 and $31 \%$. Interestingly, different digital procedures are not introduced at the same time. This is not surprising since digital indexing is required for digitisation; however, it also indicates that the introduction of new technologies is a stepwise process, which gradually involves more complex practices. The adoption of digital technologies on the management of archives is slightly lower $(55.00 \%$ of collections are already indexed and $12.81 \%$ are digitised) and more heterogeneous than overall average in terms of indexing. ${ }^{12}$

As far as Italy is concerned, only five (anonymous) archives are included in the Enumerate Core Survey 3, an even smaller sample than in Core Survey 2, which included nine Italian archives.

An extensive analysis of the actual magnitude of the use of digital technology in Italian archives is performed in the next section using a larger and more

\footnotetext{
${ }^{12}$ This is consistent with Borowiecki and Navarrete (2015)'s empirical findings based on the Enumerate Core Survey 2 data.
} 
comprehensive dataset. However, some preliminary findings can be drawn by comparing Italian data with European counterparts. According to this survey, indexing and digitisation in Italian archives are close to the sample average. With respect to the previous survey edition (Core Survey 2: $38.56 \%$ already indexed and $8.00 \%$ already digitised), Italy reduces the distance to its counterparts. However, it must be noted that Core Survey 2 included a larger number of observations.

The Italian archives' average share of collections already indexed is lower than $54 \%$ (it was $40 \%$ in Core Survey 2), and more than half of collections have to be digitised in the future. Such preliminary findings highlights that, although Italy was one of the first countries in Europe in developing digital projects, the actual extent of the adoption of such technologies in archives is lower than other European countries. The question is to ascertain whether such a gap is homogeneous or depends on the digital divide that characterizes Italy. To analyse this issue the next section will present results drawn from an original survey conducted on Italian PHAs as well as on data on digital access to Italian PHAs' websites. The extent of digital consumption (digital access) is reported in Table 2. Again, apart from the substantial heterogeneity in Europe, only offline procedures for digital access have been developed so far, while online access is still at the beginning. Italy shows, in this case, levels of provision in line with the European average.

\section{Use and Drivers of Digital Technologies Diffusion: A Survey of Italian Public Historical Archives}

As previously illustrated, digital technologies can be applied for different purposes, and to a different extent in the preservation and utilisation of cultural goods. The range goes from: the use of personal computers for administration purposes; to the application of the most advanced photographic technologies in order to obtain high resolution; to digital scans of paintings and drawings; or to 3D virtualisation of archaeological sites; or to the use of advanced software for in-time data collecting data and monitoring.

In this section, we focus on two specific applications of these technologies in Italian public historical archives: the use of internet websites; and the digitisation of documents. These two applications are of primary importance in the context of conservation, preservation and utilisation of collections held by PHAs. The use of a website guarantees publicity of basic information (opening times, address, provided services, index of preserved material) and prompts the diffusion of advanced services, including digital access. Digitisation of documents prompts the development of the abovementioned national and European-level projects (Culturaltalia, Europeana, etc). To analyse the scope of these two applications we conducted an empirical analysis for PHAs operating in Italy by using different data sources: data on physical access and PHAs characteristics was drawn from the Sistema Statistico Nazionale (SISTAN) that include official statistics; data on the year of foundation of PHAs' websites was drawn from the Internet Archive-Wayback Machine, a web repository including snapshots of websites and by browsing archives' websites; 


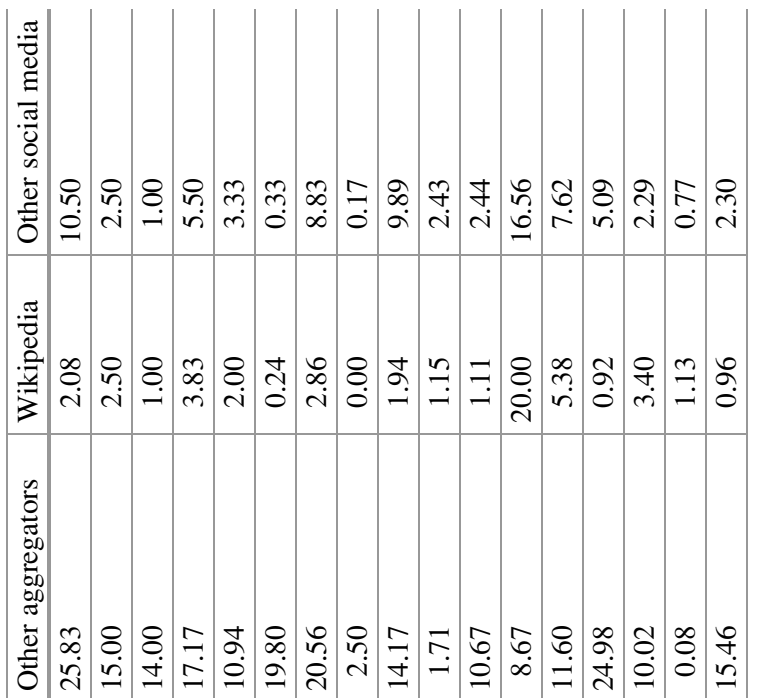

$\stackrel{\varpi}{\tilde{\pi}}$

苛 馬

$\frac{5}{}$

कू.

.ี ż

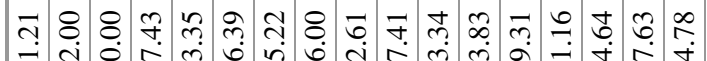
萬

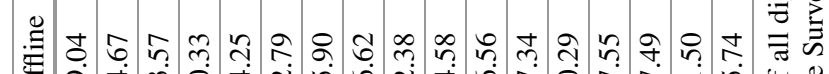

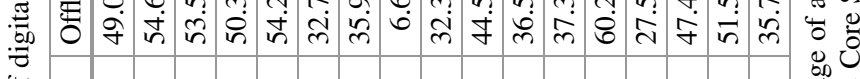

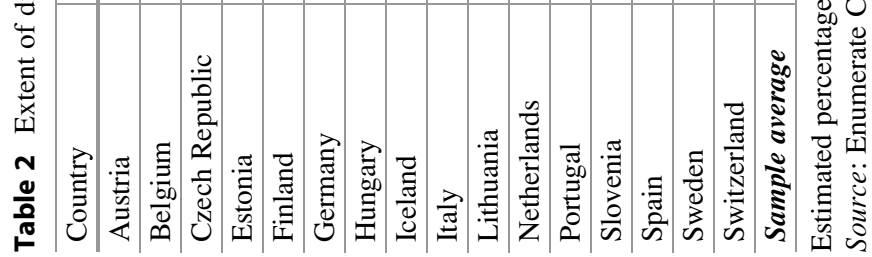


data on the use of internet was drawn from access statistics of all available Italian websites ( 83 websites in 2013), provided by the MiBACT; data for the analysis on digitisation was drawn from an original survey of 31 PHAs and local sections operating in Italy. The survey was carried out in 2014 and targeted managers of all Italian PHAs. Although the sample is larger than Enumerate, it is still partial and all findings reported have to be considered cautiously. ${ }^{13}$ This survey provides information on the characteristics of PHAs (i.e. size, type of activity, location), typology of digital project, as well as on how decisions eventually leading to adoption were made and so on.

\subsection{Some Preliminary Findings}

We start by showing general data on PHAs (Table 3), which indicates relevant differences at the regional level in terms of dimension, thus confirming structural geographical differentiation within the country (data reported in relative terms, that is, per PHA): in general, PHAs located in the North and in the Centre of Italy are larger in terms of surface area and shelving provision, but have, on average, a lower number of workers. At the same time, the number of items per inhabitant varies across regions, showing the highest value in the Centre. ${ }^{14}$ An analogous geographical divergence emerges on the demand side by comparing the number of visitors and consultations and these are considerably lower in the South. The average values for the number of years since a website has been used seem, conversely, to deny the presence of a strong digital divide on the supply side, although the average value, in this case, hides a very large variability in the sample.

\subsection{The Use of Internet Websites}

We used data drawn from website statistics to analyse the extent of the use of websites by Italian PHAs. The dataset included information on all the available PHAs websites in the MiBACT's web domain in the period 2010-2013 and several PHAs websites with different domains. Although websites are a low cost technology which spread very fast in the last decades, relatively few PHAs used them in 2010. In fact, in 2012, the MiBACT undertook several projects to support the adoption of digital technologies, including the usage of websites by PHAs. The large majority of websites have been then included in the MiBACT's domain,

\footnotetext{
${ }^{13}$ We thank the General Direction for Italian Archives for the support in the collection of the data used in Sect. 4.

${ }^{14}$ Items include, in this Table, the number of manuscripts and documents, which represent the core of Italian archives' collection and provide a measure of the quantity of objects preserved by such institutions. PHAs conserve also negatives, microfilms, pictures, etc. and several copies and backups of the same item, which we do not consider in order to avoid biased evaluations.
} 


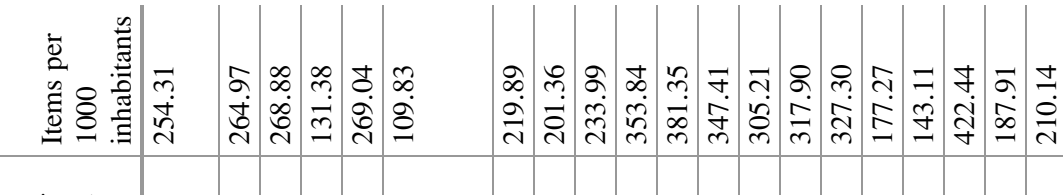

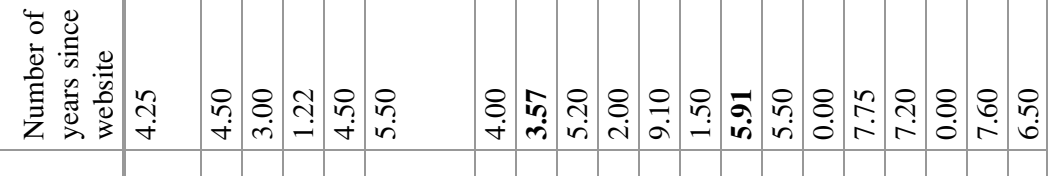
$\stackrel{0}{0}$

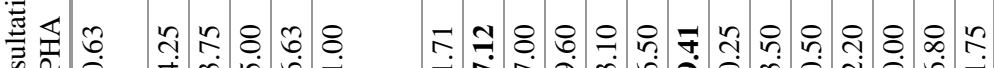

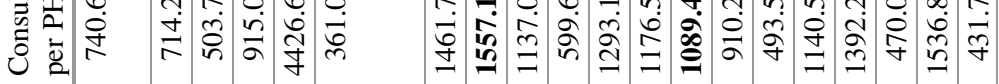

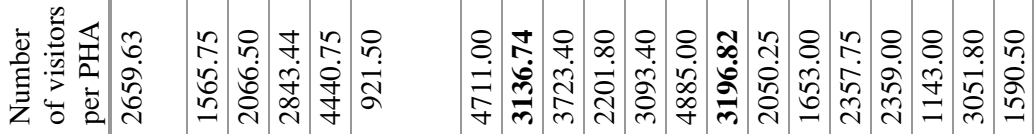

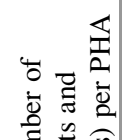

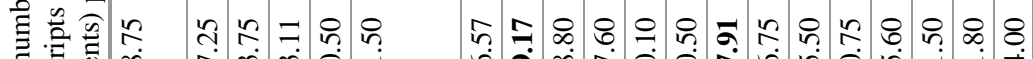

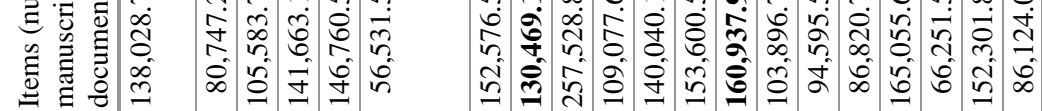

$\bar{\Xi}$

పัต 离苛

莺

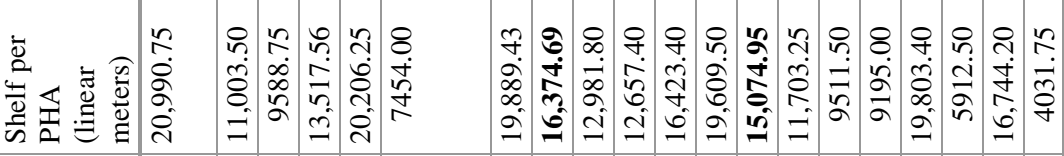

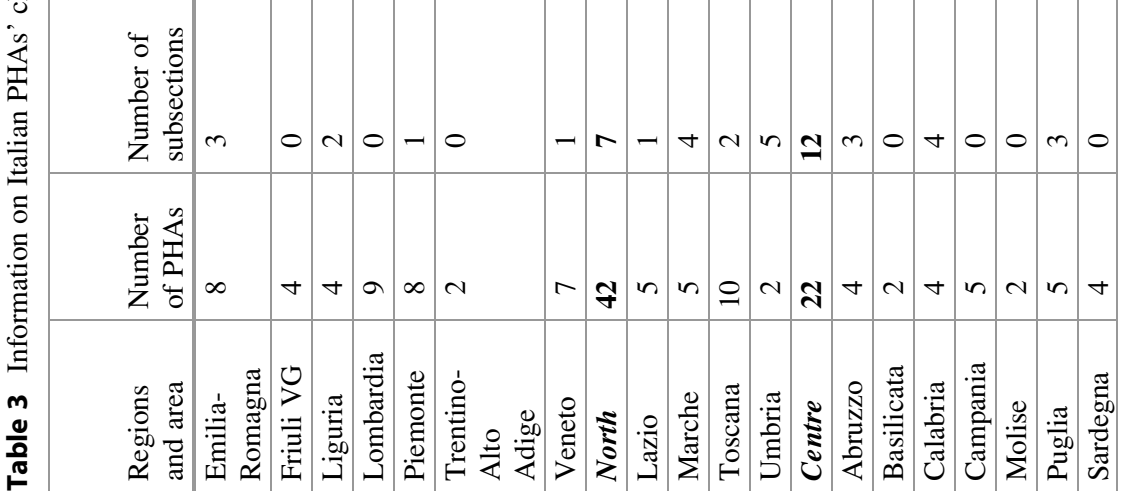




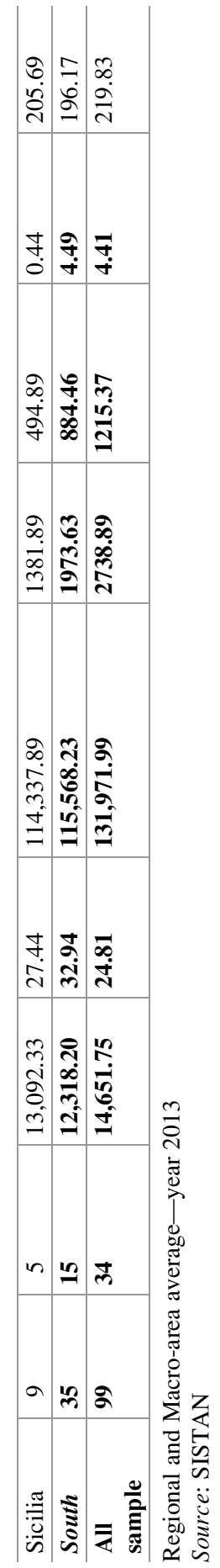


Fig. 2 Percentage of PHAs having websites. 2010-2013. Source: our computation

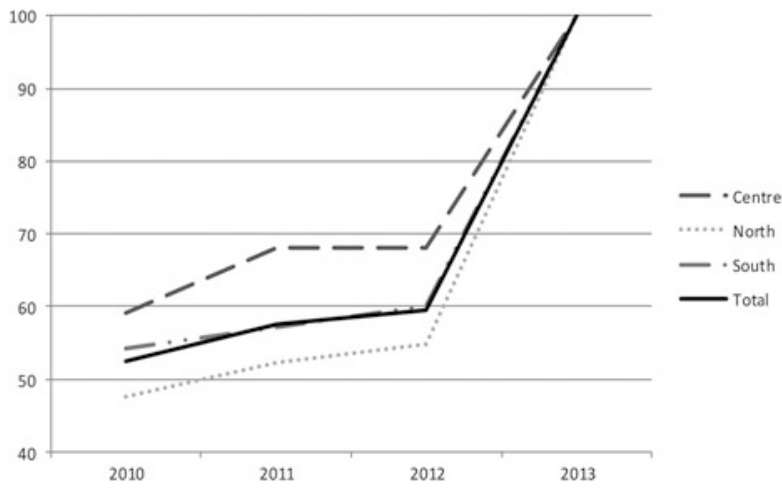

beniculturali.it. As a consequence, the number of PHAs using website dramatically increased after 2012 (Fig. 2). ${ }^{15}$

However, the presence of a website is only a rough measure of the use of digital technologies for at least two reasons: it does not say anything about the extent of digitisation or digital indexing; and a website can be used to provide a potentially wide range of services, from general information on the archive (address, opening times, etc.) to the direct provision of services such as digital access. In fact, strong geographical differences emerge in the website usage as shown in Fig. 3, which displays the number of website visitors per PHA in the three areas in 2013: visitors are defined as uniquely identified client (IP) who accessed at least a page in that period. Although it represents a demand-side measure, it should be noted that it depends strictly on the amount and quality of pages and services provided by the website.

As previously mentioned, digital projects undertaken by European and national institutions aim to enhancing universal access to cultural goods, through increasing physical and digital access. To analyse whether the introduction of digital technologies has been effective in this sense we look at the dynamics of physical (Fig. 4) and digital (Fig. 5) access in Italian archives.

We use four measures of physical access: number of presences, number of for-studying and not-for-studying consultations and number of archival groups consulted; and two measures of digital access: the abovementioned number of visitors and the number of visits, the latter referring to visitors accessing at least a page and who did not access other website pages in the previous $60 \mathrm{~min}$. Comparing Figs. 4 and 5, it appears that physical access did not change notably while digital access increased dramatically in total values. One may claim that such dynamics imply a more diffuse access to cultural products preserved by Italian PHAs. However, the reader should be reminded that online access to cultural material is still rather limited. Therefore, the results could be due to the increasing number of websites rather than an increasing supply of digitised material.

\footnotetext{
${ }^{15}$ Note that the number of PHAs did not change in this interval.
} 


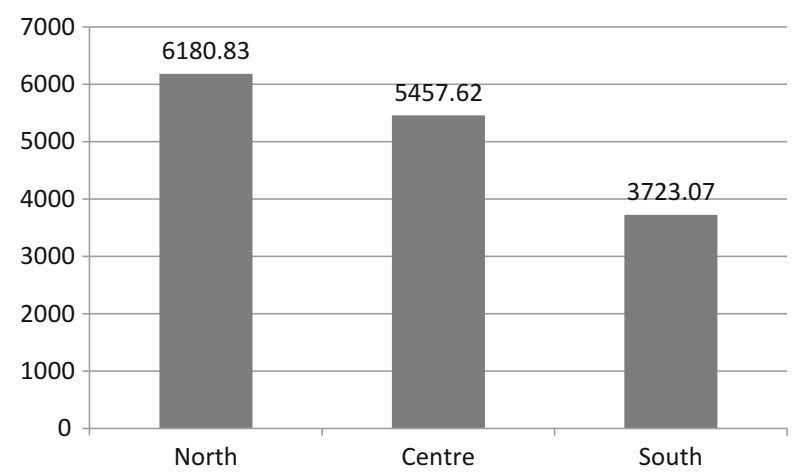

Fig. 3 Website visitors per PHA-PHA average value per area-2013. Source: our computation on websites' access statistics

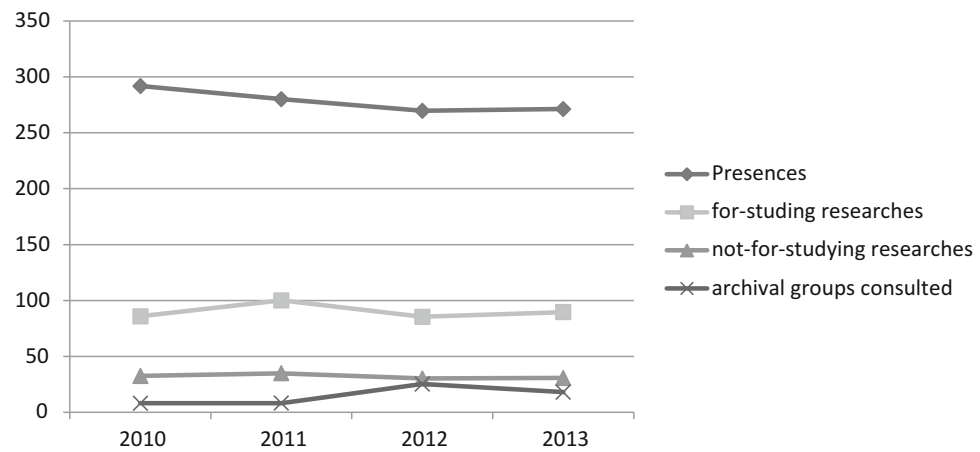

Fig. 4 Physical access-2010-2013-Total values in thousands. Source: our computation on SISTAN data

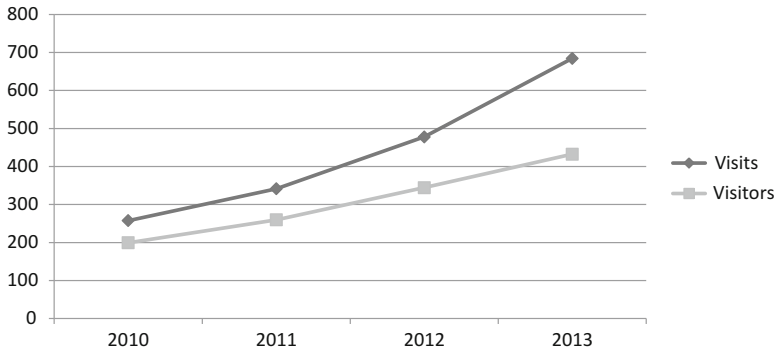

Fig. 5 Digital access-2010-2013-Total values in thousands. Source: our computation on websites' access statistics 
Fig. 6 Digitisation and digital access. Percentage by area. Source: our computation

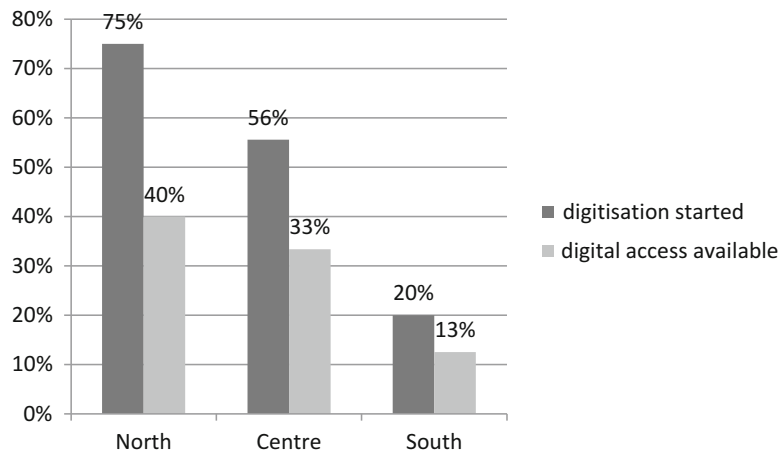

\subsection{The Extent of Digitisation in Italian Archives}

To investigate the actual scope of content digitisation in Italian archives, we use data drawn by the original survey that we conducted in 2014 that includes 31 observations. Respondents are quite homogenously distributed in the three geographical macro-areas and represent $23 \%$ of PHAs and local subsections in Italy ( $24 \%$ of the PHAs in the North, $26 \%$ of those located in the Centre, and $20 \%$ of those in the South). Figure 6 shows the percentage of PHAs that started a process of digitisation and allows for digital access online as area percentage. The adoption of digital technologies in Italian PHAs clearly appears not to be homogeneous between these areas: digitisation reaches $75 \%$ in the Northern area but digital access is still very limited in the country overall.

A digital divide therefore exists in the provision of digital services and, more significantly, in the progress that PHAs have made in starting the process of digitisation of the items they preserve. Note that this is consistent with previous findings on geographical differences across areas in website visits (Fig. 3). At the same time differences also occur in physical access (see columns seven and eight in Table 3). Not only the quality of PHAs collections and the extent of their digitization but also the education level, income and social capital are relevant to explain the above differences.

\section{Conclusions}

This chapter highlights several aspects concerning the introduction of digital technologies in the management of Italian PHAs and in the conservation, preservation and utilisation of their cultural heritage. From a theoretical point of view, the characteristics of PHAs raise interesting questions regarding the definition of efficiency condition transforming a rival and excludible good into a potentially pure public good. An additional important issue is whether digital access is either a complement or substitute to the real one. 
Here we also investigate issues related to ICT for Italian PHAs. The analysis does not allow us to draw clear-cut conclusions because of the quantity and the quality of available data but, nevertheless, some tentative conclusions can be drawn. In general, the spread of ICT in European cultural institutions is still limited although the first projects started several years ago and several programs at continental and national level have been launched since then. The absence of an adequate system of incentives may help to explain the slow advance in the production of metadata by cultural institutions and their provision to national aggregators and from them to Europeana. Moreover, from a different perspective, recent severe budget constraints in the public sectors in the EU may have played a relevant role in slowing down ICT implementation that, conversely, would require substantial investments. Furthermore, the fragmentation of available resources across several programs, not always sufficiently coordinated, may undermine their effectiveness.

The impact of the abovementioned issues is likely to be even more critical if we consider the peculiarities of the ICT implementation. In fact, our analysis highlights that the introduction of ICT is a long-term stepwise process involving the coordination of several actors operating in different institutions and levels. This is particularly true for PHAs, which were shown to be resistant to adapting their practises to a changing environment of ICT. Regarding this issue, we find that only basic technologies, such as indexing, have been introduced in the management of PHAs while more complex advancements, such as digitisation and on-line access are still at a preliminary stage. This happened in Europe as much as in Italy, where the MiBACT supported the spread of ICT in PHAs, leading mainly to the general adoption of some unsophisticated practices, such as basic websites.

However, Italy is characterized by considerable geographical differences in supply and demand. Differences emerge on the demand side, in terms of number of visits and visitors to archives' websites. This might be just partially connected with geographical gaps in economic and social conditions, with a relevant role played by human capital accumulation, but also with the differences in the provision of digital services and in the extent of digitisation of PHAs' collections. These differences call for enhancing the effectiveness of the existing programs and strengthening the system of incentives toward digitisation. Furthermore, consumption of digital services has not increased substantially whereas the intensity of usage has indeed grown, mainly because of the proliferation of websites. As for physical consumption, this stays virtually unaffected. The fact that digital services are yet to be developed in a meaningful way does not allow us to draw conclusions on the relationship between physical and digital utilisation for Italian archives.

As a final point we would stress the importance of data collection as a tool for monitoring the progress in the implementation of ICT in the field of cultural heritage management. As ICTs requires a radical change in practises and considerable investments, policy-makers need to have complete and up-to-date information to fine-tune policies and develop effective programs. The limited participation to Enumerate, even reduced in the last edition, suggests that voluntary provision of data is not effective, at least in the absence of a system of incentives. This calls for incorporating data collection in the design of new programs to guarantee a complete flow of information during the implementation stage. 
Open Access This chapter is distributed under the terms of the Creative Commons AttributionNoncommercial 2.5 License (http://creativecommons.org/licenses/by-nc/2.5/) which permits any noncommercial use, distribution, and reproduction in any medium, provided the original author(s) and source are credited.

The images or other third party material in this chapter are included in the work's Creative Commons license, unless indicated otherwise in the credit line; if such material is not included in the work's Creative Commons license and the respective action is not permitted by statutory regulation, users will need to obtain permission from the license holder to duplicate, adapt or reproduce the material.

\section{References}

Bakhshi, H., \& Throsby, D. (2012). New technologies in cultural institutions: Theory, evidence and policy implications. International Journal of Cultural Policy, 18(2), 205-222.

Borowiecki, K. J., \& Navarrete, T. (2015). Digitization of heritage collections as indicator of innovation. University of Southern Denmark, Discussion Papers on Business and Economics No. $14 / 2015$.

Caffo, R. (2014). Digital cultural heritage projects: Opportunities and future challenges. Procedia Computer Science, 38, 12-17.

Clough, G. W. (2013). Best of both worlds: Museums, libraries, and archives in a digital age. Washington, DC: Smithsonian Institution.

Di Giorgio, S. (2014). Culturaitalia, the Italian national content aggregator in Europeana. Procedia Computer Science, 38, 40-43.

European Commission. (2011). Commission recommendation of 27 October 2011 on the digitisation and online accessibility of cultural material and digital preservation. Official Journal of the European Union, 54.

Flew, T., \& Swift, A. (2013). Cultural policy. In R. Towse \& C. Handke (Eds.), Handbook on the digital creative economy (pp. 155-161). Cheltenham, England: Edward Elgar.

Giardina, E., Mazza, I., Pignataro, G., \& Rizzo, I. (2015). Voluntary provision of public goods and technology. Paper presented at the International Atlantic Economic Conference, Milan, Italy, March 11-14.

MiBACT. (2014). Culture in Italy - Basic figures 2014. Roma: Gangemi Editore.

MiBACT. (2015). Retrieved November 9, 2015, from http://www.beniculturali.it/mibac/export/ MiBAC/sito-MiBAC/MenuPrincipale/Progetti/index.html

Nauta, G. J., \& van den Heuvel, W. (2015). Survey report on digitisation in European Cultural Heritage Institutions 2015. Europeana/ENUMERATE, DEN Foundation (NL). Retrieved from http://pro.europeana.eu/files/Europeana_Professional/Projects/Project_list/ENUMER ATE/deliverables/ev3-deliverable-d1.2-europeana-version1.1-public.pdf

Navarrete, T. (2013a). Digital cultural heritage. In I. Rizzo \& A. Mignosa (Eds.), Handbook on the economics of cultural heritage (pp. 251-271). Cheltenham, England: Edward Elgar.

Navarrete, T. (2013b). Museums. In R. Towse \& C. Handke (Eds.), Handbook on the digital creative economy (pp. 330-343). Cheltenham, England: Edward Elgar.

Paolini, P., Mitroff Silvers, D., \& Proctor, N. (2013). Technologies for cultural heritage. In I. Rizzo \& A. Mignosa (Eds.), Handbook on the economics of cultural heritage (pp. 272-289). Cheltenham, England: Edward Elgar.

Peacock, A. (2006). The arts and economic policy. In V. A. Ginsburgh \& D. Throsby (Eds.), Handbook of the economics of art and culture (Vol. 1, pp. 1124-1140). Amsterdam: North Holland.

Peacock, A., \& Rizzo, I. (2008). The heritage game. Oxford, England: Oxford University Press. 
Salaün, J.-M. (2013). The immeasurable economics of libraries. In I. Rizzo \& A. Mignosa (Eds.), Handbook on the economics of cultural heritage (pp. 290-305). Cheltenham, England: Edward Elgar.

Stroeker, N., \& Vogels, R. (2014). Survey report on digitisation in European cultural heritage institutions 2014. ENUMERATE thematic network, Panteia (NL). Retrieved from http://www. enumerate.eu/fileadmin/ENUMERATE/documents/ENUMERATE-Digitisation-Survey-2014. pdf

Vicente, M. R., \& Lopez, A. J. (2011). Assessing the regional digital divide across the European Union-27. Telecommunication Policy, 35(3), 220-237. 